\title{
SIMULACIÓN Y ANÁLISIS DEL COMPORTAMIENTO TÉRMICO DE UNA SALA INFORMÁTICA EN UNA ZONA CÁLIDO-HUMEDA
}

\author{
SIMULATION AND ANALYSIS OF TE THERMAL BEHAVIOR OF \\ COMPUTER ROOM IN A WARM-HUMID ZONE
}

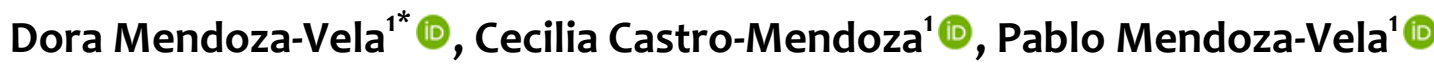

${ }^{1}$ Sede Regional Orán de la Universidad Nacional de Salta, Ciudad de Orán, Prov. de Salta, Rep. Argentina

Recibido (Recieved): 21/02/2020 Aceptado (Accepted): 05/03/2020

\section{RESUMEN}

El objetivo de este trabajo es conocer el comportamiento térmico de una Sala Informática en diferentes situaciones de uso académico. La misma se encuentra en una Sede Universitaria de la ciudad de Orán (Provincia de Salta, Argentina), lugar caracterizado por tener un clima cálido-húmedo. La simulación se realiza para un periodo de 11 días dentro el periodo lectivo, recurriendo a los datos del Servicio Meteorológico Nacional y a mediciones in situ. El software usado para la simulación es SIMEDIF. La metodología de trabajo incluye actividades de relevamiento, mediciones, preparación de datos y de escenarios académicos, simulación y análisis de resultados. A partir de los resultados de la simulación se detectan situaciones de disconfort y se calcula la carga térmica requerida para superarlas. Se plantea la necesidad de incorporar estrategias bioclimáticas y de enfriamiento pasivo a fin de lograr el confort higro-térmico con el mínimo consumo energético y el mínimo impacto al medio ambiente. La importancia del trabajo radica en poder lograr una primera aproximación al comportamiento real térmico de un ambiente mediante la modelización y simulación con herramientas computacionales.

Palabras Clave: Simulación, SIMEDIF, Comportamiento térmico, Zona cálido-húmeda, Confort térmico.

\section{ABSTRACT}

The objective of this paper is to know the thermal behavior of a computer room in different class. It is located at University Headquarters in the city of Orán (Province of Salta, Argentina), a place characterized by having a warm-humid climate. The simulation is carried out for 10 days and coincides with the class period, using data from National Meteorological Service and on-site measurements. The simulation software used is SIMEDIF. The work methodology includes recognition activities, measuring, data and scenes preparation, simulation and analysis of results. From the results obtained, uncomfortable situations are detected and thermal load required to overcome them is calculated. It is necessary to incorporate bioclimatic and passive cooling strategies in order to achieve hygro-thermal comfort with minimum energy consumption and minimum environmental impact. The importance of the work lies in obtaining a first approximation to the real thermal behavior of an environment through modeling and simulation with computational tools.

Keywords: Simulation, SIMEDIF, Thermal behavior, Warm-humid zone, Thermal comfort.

\footnotetext{
* Corresponding author:

E-mail:dmendoza412@gmail.com
} 


\section{INTRODUCCIÓN}

\subsection{EL CONFORT TÉRMICO}

La idea de confort ha evolucionado de manera que en distintos periodos ha asumido diferentes significados. Según la American Society of Heating, Refrigerating and Air-Conditioning Engineers (ASHRAE), el confort "es la condición mental que expresa satisfacción con el entorno". Algunos factores a considerar son la temperatura, la humedad, la iluminación, los ruidos, los vapores, los olores, la presencia de humos y los grados de polución atmosférica.

Según la norma ISO 7730 el confort térmico "es una condición mental en la que se expresa la satisfacción con el ambiente térmico". Depende de varios parámetros globales externos, como la temperatura del aire, la velocidad del viento y la humedad relativa, y otros específicos internos como la actividad física desarrollada, la cantidad de vestimenta 0 el metabolismo de cada individuo. Según Beccali et al [1], este concepto suele ser subjetivo, cada persona es diferente y sus percepciones individuales pueden diferir ampliamente. Mediante técnicas experimentales y valoraciones subjetivas se han realizado estudios sobre los márgenes de los factores climáticos en los cuales la gran mayoría de las personas se encuentran cómodas.

\subsection{LOS EDIFICIOS, EL CONSUMO ENERGÉTICO Y LA EMISIÓN DE GASES DE EFECTO INVERNADERO}

En las sociedades actuales, la disponibilidad de energía está fuertemente ligada al nivel de bienestar, salud y duración de vida del ser humano. Los siglos XX y XXI se caracterizan por una demanda creciente de energía a nivel global. La población urbana mundial viene creciendo sostenidamente en los últimos siglos, debido al incremento demográfico propio y a la fuerte migración de las poblaciones de zonas rurales a las grandes ciudades. Según el informe de la ONU [2], para el año 2014, el 54\% de la población mundial habitó en zonas urbanas, para el año 2018, ese valor se incrementó a 55,3\%.

Los edificios proporcionan un entorno de vida y de trabajo a los seres humanos: el $80 \%$ de la vida de las personas transcurre en el interior de los edificios. Durante el año 2004 solamente los edificios agotaron casi el $37 \%$ de la energía del mundo, y se proyecta que alcance el 42 \% para el año 2030. En Europa, en el año 2000 , el $45 \%$ de la energía producida se usó en el sector edilicio y el $50 \%$ de la polución fue causada por el mismo sector [3].
El consumo edilicio está fuertemente relacionado con el comportamiento de los habitantes, cuyas demandas energéticas están sujetas a las temperaturas ambientales exteriores. En Argentina, el consumo eléctrico suele incrementarse en un $50 \%$ más en época de verano, y el consumo de gas residencial, hasta 8 veces en épocas frías. Es así que más del 50\% del consumo eléctrico y de gas se llevan los edificios, valores que podrían reducirse hasta un $76 \%$ si se aplicaran políticas efectivas de fomento a la eficiencia energética y de planificación urbana [4].

Los edificios tienen una participación importante en las emisiones de $\mathrm{CO}_{2}$ : durante la producción de los materiales y transporte, durante su construcción y a lo largo de su vida útil debido al uso de gas, electricidad y carbón, entre otros, para cocina, agua caliente, iluminación, refrigeración y calefacción de ambientes. Resulta sumamente preocupante el panorama de emisiones actuales y futuras de los edificios en distintas zonas del mundo para el año 2030 (Fig. 1). En el caso de América Latina, prácticamente se duplicarán sus emisiones en 20 años [5]-[6].

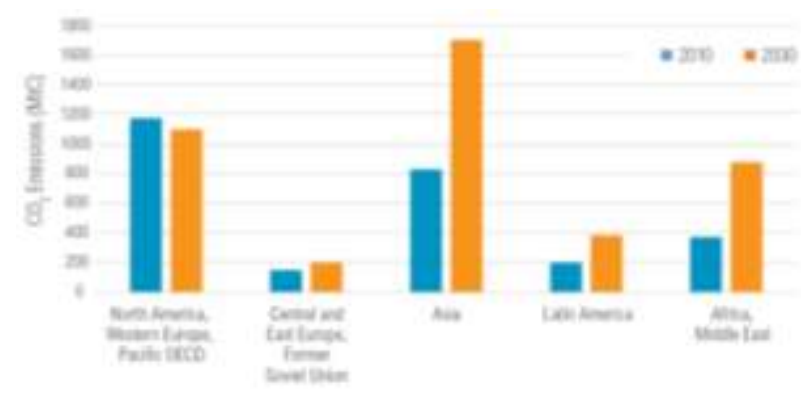

Fig. 1. Emisiones actuales y proyectadas de $\mathrm{CO}_{2}$ por región mundial. [6]

\subsection{EL CONTEXTO REGIONAL DEL OBJETO DE ESTUDIO}

La ciudad de Orán se ubica en el sector central del extremo norte de la provincia de Salta, a $33 \mathrm{~km}$ del Trópico de Capricornio y a $50 \mathrm{~km}$ de la frontera con la República de Bolivia. Sus coordenadas son $23^{\circ} 08^{\prime} 10^{\prime \prime}$ latitud S y $64^{\circ} 19^{\prime} 20^{\prime \prime}$ longitud O, a $336 \mathrm{msnm}$. Se encuentra emplazada en el Valle de Zenta, rodeada de cerros pertenecientes a la Cordillera Oriental o precordillera de los Andes.

Según la clasificación de Koppen su clima es del tipo CWa (clima subtropical con invierno seco y verano cálido [7]. De acuerdo a la zonificación bioclimática de la Argentina (Norma IRAM 11603), Orán se encuentra en la zona Ilb (clima cálido-húmedo). Presenta periodos estivales muy calurosos, con temperaturas, humedades y precipitaciones elevadas. Los inviernos suelen tener temperaturas agradables durante el día y noches frías. La ciudad dispone de una estación 
meteorológica denominada ORAN AERO N 87016, que brinda diariamente los datos al Servicio Meteorológico Nacional (SMN).

De acuerdo a información estadística para el periodo 1981-2010 [8]-[10]: el periodo estival va desde octubre a marzo con temperatura máxima media de $31,5^{\circ} \mathrm{C}$, valores medios mensuales de humedad relativa entre $59 \%$ y $83 \%$, velocidad de viento entre 6,2 y 9,6 $\mathrm{km} / \mathrm{h}$ con un $56 \%$ mayormente proveniente del Este. Algunas temperaturas máximas absolutas que ilustran el clima actual de Orán en periodo lectivo: $41,5^{\circ} \mathrm{C}$ para setiembre de $2018,40^{\circ} \mathrm{C}$ para octubre de $2018,40^{\circ} \mathrm{C}$ para setiembre de $2019,43,8^{\circ} \mathrm{C}$ y $44,5^{\circ} \mathrm{C}$ para 28 y 29 de octubre 2019, respectivamente.

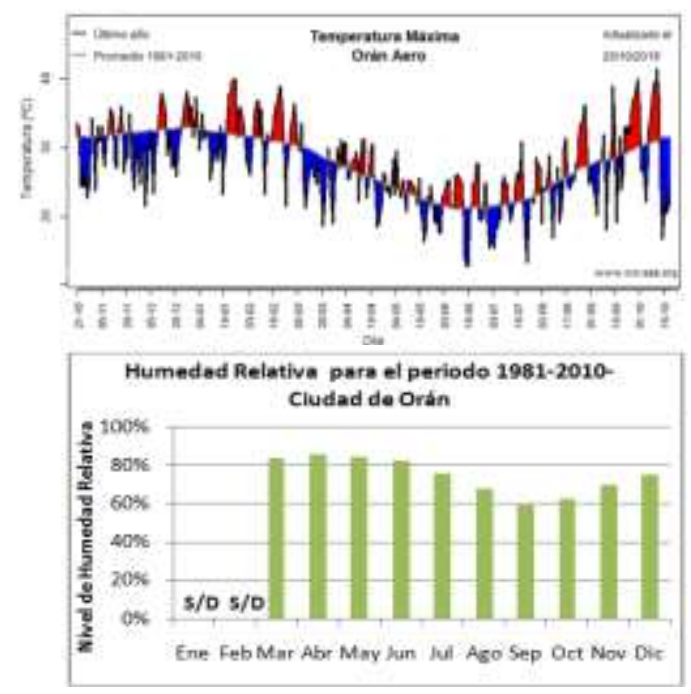

Fig. 2. Temperaturas máximas del último año (octubre de 2018 a octubre de 2019) e Información Estadística sobre temperatura y humedad relativa para el periodo 1981-2010, Ciudad de Orán. [8]-[9]

Por su latitud y condiciones climáticas, el recurso solar está disponible prácticamente todo el año. Para Orán, los valores estimados de radiación solar global acumulada anual sobre plano horizontal rondan entre 1700 y $1800 \mathrm{kWh} / \mathrm{m}^{2}$ año con valores mensuales promedios de entre $2,31 \mathrm{kWh} / \mathrm{m}^{2}$ para junio y 6,83 $\mathrm{kWh} / \mathrm{m}^{2}$ para diciembre [11].

\subsection{ASPECTOS ACADÉMICOS, ESTRUCTURALES Y FUNCIONALES DE LA SALA INFORMÁTICA DE LA SEDE UNIVERSITARIA}

La Sede Regional de Orán, dependiente de la Universidad Nacional de Salta se ubica en el centro de la ciudad. Durante los años 20 y 30 el edificio fue construido para el funcionamiento del primer hospital de la ciudad. Recién en la década del 80 fue cedido para el funcionamiento de la primera sede universitaria en la región. Desde entonces se hicieron modificaciones estructurales importantes acordes a las nuevas necesidades funcionales: sector administrativo, laboratorios, talleres, aulas, boxes docentes, salón auditórium, biblioteca, salas informáticas.

Actualmente, la Sede ofrece carreras de las áreas de Salud, Exactas y Naturales. Dispone de 2 salas informáticas: la primera es la de mayor capacidad (22 personas) y está destinada a todas las carreras, la segunda es más reducida y está destinada únicamente a las carreras del área de informática. Por lo general, ambas salas se usan de 8 a 13 y de 15 a 21 hs, de marzo a junio y de agosto a noviembre. El objeto de estudio de este trabajo es la Sala mayor, a la que se referenciará de aquí en más como "Sala".

Las características constructivas de la Sala son los siguientes:

- Ubicación: E-O, sobre las medianeras Norte y Este con terrenos vecinos

- Aberturas y puertas: una ventana y una puerta ambas orientadas al Sur

- Dimensiones: 6,54 x 9,87 m, su altura: 3,09 m

- Envolvente vertical: de $20 \mathrm{~cm}$ de ladrillo macizo, con revoque interior y exterior

- Paredes internas y externas: 70\% pintadas de blanco, y el resto de color beige en su parte inferior

- Techo: chapa de zinc + cámara de aire de $50 \mathrm{~cm} \mathrm{+}$ cielorraso de poliestireno expandido de $2 \mathrm{~cm}$

- Piso: contrapiso + cerámico gris claro

- Carpinterías: de madera, con infiltraciones.

La Sala limita con dos terrenos vecinos mediante las paredes Norte y Este y está parcialmente sombreada por árboles tanto por el Este como por el Norte. Hacia el Oeste limita con otra aula amplia.

El uso de la Sala es variado, por ello se conforman diferentes escenarios académicos: uso total, parcial o nulo de los equipos informáticos. Está equipada con 22 computadoras tipo PC, un rack con equipos para la comunicación y distribución de red e internet (switchs y routers), equipo multimedial (proyector y pantalla). El $40 \%$ de las PCs son de última generación, mientras que las restantes tienen componentes actualizados. EI $100 \%$ de las computadoras tiene software libre $y$ programas específicos para las diferentes cátedras. Estos datos son necesarios para estimar el calor que disipan los equipos informáticos cuando están en funcionamiento.

Para el logro del confort térmico en días calurosos se dispone de un equipo de aire acondicionado de 15000 Frigorías.

\section{ANTECEDENTES}

Los programas computacionales de simulación constituyen una herramienta altamente valiosa, 
especialmente en entornos de desarrollo tecnológico. Permiten crear modelos de hechos y/o procesos de manera rápida, eficiente y a bajo costo, proponer diferentes configuraciones, modificar parámetros y ver el comportamiento, trabajar con sistemas complejos (que serían difíciles de abordar con métodos analíticos), con situaciones peligrosas (a fin de evitar daños) o de altos costo de implementación en contextos reales. Los resultados de toda simulación contribuyen a la toma de decisiones en el sector que corresponda.

En el caso de los edificios, la utilización de herramientas de simulación se puede aplicar a varios aspectos, entre ellos, su relación con la energía. Conocer de antemano este comportamiento contribuirá en la toma de decisiones durante el diseño, o bien si se trata de un edificio ya existente, durante el reacondicionamiento en pos de confort y reducción del consumo energético.

Entre las herramientas más conocidas para la simulación térmica se encuentran Energy Plus y SIMEDIF.

SIMEDIF es un software gratuito desarrollado en el Instituto de Investigaciones en Energía no Convencional (INENCO, UNSa-CONICET, Salta, Argentina). Puede descargarse libremente de internet [12]. La versión original data del año 1984, fue diseñada para plataforma DOS por los Dres. Graciela Lesino, Luis Saravia y Dolores Alía. La versión para Windows fue desarrollada por la Dra. Silvana Flores Larsen. El software ha sido validado con datos experimentales a través de los años por investigadores argentinos y del exterior. Cuenta con usuarios de Latinoamérica y España y se enseña además en diversas maestrías, doctorados y licenciaturas de Argentina y Uruguay. Existen varios artículos científicos que han hecho uso de esta herramienta [13]-[21].

\section{METODOLOGÍA}

La simulación se realizó para un periodo de 11 días e incluyó las siguientes actividades:

- Relevamiento de la geometría de la sala, actividades académicas realizadas y horarios

- Relevamiento de las características del equipo de refrigeración y tiempo de uso

- Relevamiento de las necesidades de confort térmico durante las clases

- Obtención de datos oficiales de temperatura y humedad relativa de la ciudad

- Medición de datos de radiación de la ciudad

- Configuración de escenarios y carga de datos en SIMEDIF

- Simulación de escenarios

- Análisis de los modelos generados.
Los datos de temperatura y humedad relativa se obtuvieron del SMN correspondiente al periodo simulado. Debido a que la estación del SMN no cuenta con mediciones de radiación solar, se midió en forma manual la radiación global sobre superficie horizontal en el predio de la Sede, de 11 a 15 hs, con intervalos de 15 minutos. Los datos faltantes se completaron utilizando la herramienta Geosol [22] y el Método de Liu Jordan.

El recurso de simulación usado fue SIMEDIF.

\subsection{RECURSO DE SIMULACIÓN: SIMEDIF}

SIMEDIF ha sido concebido como una herramienta de diseño para calcular la temperatura horaria del aire dentro de los espacios de un edificio, la temperatura superficial horaria de las paredes y la energía auxiliar de calentamiento/enfriamiento que se necesita para mantener los espacios a una temperatura determinada por un termostato que puede definirse hora por hora para el año completo. El modelo de cálculo se encuentra descripto en el trabajo de Flores Larsen y Lesino [13]. También cuenta con un módulo para calcular las condiciones de confort dentro de los espacios, mediante el método del Voto Medio Predicho (PMV) y el Porcentaje de Personas Insatisfechas(PPD), según la norma ASHRAE 55. Además permite la adición de sistemas pasivos e híbridos como enfriadores tierra-aire, enfriadores evaporativos, doble fachadas verdes, etc. Por ello cuenta con diferentes bases de datos: de materiales y sistemas constructivos, de plantas para las fachadas verdes, de archivos meteorológicos anuales para diversas localidades en Argentina y Sudamérica, como por ejemplo:

- Base de datos de materiales según la Norma IRAM

- Sistemas constructivos predefinidos con materiales locales y posibilidad de definir materiales de la zona

- Base de datos de archivos meteorológicos para Argentina (en formato EPW compatibles con Energy Plus) incluidos en la instalación y posibilidad de usar archivos climáticos de otras regiones de Latinoamérica y el mundo mediante descarga de los sitios web que disponen de archivos EPW

- Modelos de estimación de temperatura horaria para climas secos en base a valores medios diarios.

La versión actual cuenta con dos partes: la interfase para el ingreso de datos, codificada con el Lenguaje de Programación Visual Basic 6.0, y el módulo de cálculo, totalmente desarrollado en Python. La entrada de 
datos es transparente (archivos de texto plano), por lo que cualquier programador puede desarrollar su propia interfase de ingreso si así lo requiere. El programa genera archivos de texto plano con los resultados calculados hora por hora (temperatura interior de las zonas, temperaturas de las paredes, ganancia auxiliar requerida, PMV/PPD, etc.). El Software incluye un módulo gráfico (DView) que permite analizar los datos por periodos, realizar promedios y análisis estadístico.

En el caso de edificios existentes, como el presentado en este trabajo, el software puede utilizarse para validar el modelo térmico de construcción a través de datos medidos, o bien, para cuantificar la eficacia de las posibles alternativas de rediseño en el caso de trabajar en rehabilitación energética [12].

Para realizar la simulación, el edificio se debe dividir en zonas conectadas térmicamente entre sí a través de diferentes elementos como paredes, muros de agua, tabiques, aberturas y vanos, ventanas y ventanillas. Para cada elemento estructural se definen sus propiedades básicas: tipo de material, dimensiones, propiedades térmicas y/u ópticas de los materiales, otros, superficies con exposición solar, otros

En cada zona térmica se deben describir las ganancias internas, provenientes de:

- estufas o refrigeración de aire con equipos de aire acondicionado (en cuyo caso los valores deben ser negativos indicando que se extrae calor)

- iluminación, solamente la parte que se disipa como calor al ambiente (que generalmente es menor que el consumo eléctrico)

- personas

- $\quad$ y otros equipos (que no entren en las categorías anteriores).

Las renovaciones de aire pueden ser voluntarias (ventilación debido a la apertura de ventanas, extractores de aire, etc.) o involuntarias (infiltraciones a través de la carpintería y/o rendijas) y se expresan en unidades de $1 / \mathrm{h}$ (cantidad de veces que se renueva completamente el aire de la zona en una hora). En el primer caso se consideran las aperturas de ventanas, los extractores de aire; el segundo caso se refiere a las infiltraciones de aire a través de rendijas y marcos, dependiendo de la calidad de la carpintería. Por razones de higiene y seguridad se debe asegurar un valor mínimo de renovaciones, usualmente reglamentado en normativas, dependiendo del número de personas en la zona y del tipo de actividad desarrollada.

\subsection{CRITERIOS PARA EL ANÁLISIS DEL CONFORT}

El análisis de la situación de confort se realizó mediante el cálculo del índice PMV (incluido en SIMEDIF) de acuerdo al modelo ASHRAE 55 (ver TABLA I) y teniendo en cuenta las siguientes condiciones:

- Índice de arropamiento: 0,5 clo

- Velocidad interior del aire: 0,1 m/s

- Tasa metabólica: 1,6 met.

TABLA I

Categorías de Confort según ASHRAE 55

\begin{tabular}{|c|c|}
\hline Categorías & Rango del PMV \\
\hline Caliente & $\mathrm{PMV}>2,5$ \\
\hline Cálido & $1,5<\mathrm{PMV}<2,5$ \\
\hline Ligeramente cálido & $0,5<\mathrm{PMV}<1,5$ \\
\hline Neutral & $-0,5<\mathrm{PMV}<0,5$ \\
\hline Ligeramente fresco & $-1,5<\mathrm{PMV}<-0,5$ \\
\hline Fresco & $-2,5<\mathrm{PMV}<-1,5$ \\
\hline Frío & $\mathrm{PMV}<-2,5$ \\
\hline
\end{tabular}

\subsection{ESCENARIOS DE SIMULACIÓN}

Usando el software SIMEDIF, y atendiendo al uso de la Sala por parte de los diferentes cursos, se configuraron 4 situaciones posibles de uso:

- Escenario base 1 (E1): sin actividad académica

- Escenario base 2 (E2): con actividad académica, iluminación completa, usando solamente los recursos didácticos básicos como proyector y la computadora del docente, con la asistencia de 22 alumnos (capacidad completa)

- Escenario base 3 (E3): con actividad académica, iluminación completa, usando los recursos didácticos básicos (proyector y la computadora del docente), 50\% de las computadoras (11) y con la asistencia de 50\% del alumnado (11)

- Escenario base 4 (E4): con actividad académica, iluminación completa, usando los recursos didácticos básicos, $100 \%$ de las computadoras y de alumnos.

También se configuró un quinto escenario (E5), que es similar al E4 pero con el funcionamiento del equipo de aire acondicionado a $24^{\circ} \mathrm{C}$ (temperatura de confort, socialmente aceptado en la zona), a fin de conocer la carga térmica auxiliar requerida para asegurar un ambiente confortable.

Finalmente se simuló un escenario-marco $X$, que representa cualquiera de los 4 escenarios bases pero funcionando en situaciones climáticas de temperaturas extremas, propias de esta zona. A tal fin se simuló para los días 27 y 28 de octubre de 2019, con temperaturas de $43,8^{\circ} \mathrm{C}$ y $44,5^{\circ} \mathrm{C}$ respectivamente [8]. 


\subsection{PROCEDIMIENTO DE SIMULACIÓN}

Para realizar la simulación se procedió de la siguiente manera:

- Carga de datos iniciales: Lugar (latitud, longitud, altura sobre el nivel del mar), periodo de simulación y datos climáticos

- Definición y carga de las zonas térmicas: volumen, renovaciones horarias y ganancias internas

- Definición y carga de materiales y propiedades de los distintos elementos estructurales (paredes, tabiques, aberturas y vidriados)

- Control del ingreso de los datos para detectar errores o informe faltante

- Simulación y generación de gráfica de resultados.

Del relevamiento efectuado se obtuvieron los datos para la carga en el SIMEDIF (Fig. 3, TABLAS II, III y IV).

Datos Periodo de simulación: 30/10-/11 del 2018

Lugar: S.R. de la Nva Orán

Zona Horaria: -3

Latitud: -23,14, Longitud: $-64,3$

Altitud (m): 336

Volumen: $198 \mathrm{~m}^{3}$

cantidad de renovaciones: 2

Ganancias internas:

- Personas: 22, estudiando de 8 a 13 y de 15 a 21 hs

- Iluminación: 6 puntos de $36 \mathrm{~W}$, en uso de 8 a 13 hs y de 15 a 21 hs

- Equipamiento: 22 PCs con $40 \%$ de monitores CRT y 60\% tipo LEDs (potencia promedio es de $250 \mathrm{~W}$ ); 1 proyector (de $450 \mathrm{~W}$ )

- Equipo de refrigeración: 1 equipo de aire acondicionado de 15000 frigorías ( $17000 \mathrm{~W}$ ), en uso de 10 a 13 y de 15 a 21 hs, de lunes a viernes.

Fig. 3. Datos básicos para la carga en SIMEDIF: periodo, lugar, renovaciones de aire, ganancias internas.

TABLA II

Descripción de paredes de la Sala (denominada como LU3)

\begin{tabular}{|c|c|c|c|c|c|c|c|}
\hline $\begin{array}{l}\text { ID. } \\
\text { PA }\end{array}$ & Pend & Azimui & $\begin{array}{l}\text { Conec } \\
\text { ta... }\end{array}$ & $\begin{array}{c}\text { Coef. } \\
\text { Conv-rad } \\
h \\
\left(\mathrm{~W} / \mathrm{m}^{2} \mathrm{~K}\right)\end{array}$ & $\begin{array}{l}\text { Sup. } \\
\text { asol. } \\
\left(\mathrm{m}^{2}\right)\end{array}$ & $\begin{array}{l}\text { Sup. } \\
\text { total } \\
\left(\mathrm{m}^{2}\right)\end{array}$ & Capas \\
\hline \multirow{2}{*}{1} & \multirow{2}{*}{$90^{\circ}$} & \multirow{2}{*}{$270^{\circ}$} & 1:LU3 & 6 & 0 & \multirow{2}{*}{19,35} & \multirow{2}{*}{ Ref1 } \\
\hline & & & 2:Ext. & 6 & 0 & & \\
\hline \multirow{2}{*}{2} & \multirow{2}{*}{$90^{\circ}$} & \multirow{2}{*}{$180^{\circ}$} & 1:LU3 & 6 & 0 & \multirow{2}{*}{29,61} & \multirow{2}{*}{ Ref1 } \\
\hline & & & 2:Ext. & 14 & 0 & & \\
\hline \multirow{2}{*}{3} & \multirow{2}{*}{$90^{\circ}$} & \multirow{2}{*}{$90^{\circ}$} & 1:LU3 & 6 & 0 & \multirow{2}{*}{19,35} & \multirow{2}{*}{ Ref1 } \\
\hline & & & 2:Ext. & 6 & 0 & & \\
\hline \multirow{2}{*}{4} & \multirow{2}{*}{$90^{\circ}$} & \multirow{2}{*}{$0^{\circ}$} & 1:LU3 & 6 & 0 & \multirow{2}{*}{29,61} & \multirow{2}{*}{ Ref1 } \\
\hline & & & 2:Ext. & 14 & 0 & & \\
\hline \multirow{2}{*}{5} & \multirow{2}{*}{\multicolumn{2}{|c|}{$0^{\circ}$}} & 1:LU3 & 6 & 0 & \multirow{2}{*}{64,5} & \multirow{2}{*}{ Ref2 } \\
\hline & & & 2:Ext. & 0,001 & 0 & & \\
\hline
\end{tabular}

Nota: Datos técnicos sobre paredes: pendiente, azimut, ambientes que conecta, absortancia solar, coeficiente convectivo-radiativo, superficie asoleada, superficie total, capas. Referencias de las capas: Ref1: "Muro Simple de $20 \mathrm{~cm}$ con Ladrillo Macizo"

Ref2: "Tierra 2m + Contrapiso de $20 \mathrm{~cm}+$ Baldosas de $2 \mathrm{~cm}$ "

TABLA III

Descripción de tabiques de la Sala

\begin{tabular}{lllllllll}
\hline ID. TA & Pend Azimut & $\begin{array}{l}\text { Conec } \\
\text { ta... }\end{array}$ & $\begin{array}{l}\text { Abs. } \\
\text { solar }\end{array}$ & $\begin{array}{c}\text { Coef. } \\
\text { Conv-rad. } \\
\mathbf{h}\left(\mathbf{W} / \mathbf{m}^{2} \mathbf{K}\right)\end{array}$ & $\begin{array}{c}\text { Sup. } \\
\text { asol. } \\
\left(\mathbf{m}^{2}\right)\end{array}$ & $\begin{array}{c}\text { Sup. } \\
\text { total } \\
\left(\mathbf{m}^{2}\right)\end{array}$ \\
\hline \multirow{2}{*}{ 1-puerta } & $90^{\circ}$ & $0^{\circ}$ & 1:LU3 & 0,2 & 6 & 0 & 6,3 \\
& & & 2:Ext. & 0,3 & 6 & 0 & \\
\hline \multirow{2}{*}{ 2-Techo } & $90^{\circ}$ & $0^{\circ}$ & 1:LU3 & 0,2 & 6 & 0 & 64,5 \\
& & & 2:Ext. & 0,6 & 14 & 30 & \\
\hline
\end{tabular}

Nota: Datos técnicos sobre tabiques: pendiente, azimut, ambientes que conecta, absortancia solar, coeficiente convectivo-radiativo, superficie asoleada y superficie total.

TABLA IV

Descripción de ventanas de la Sala

\begin{tabular}{|c|c|c|c|c|c|c|}
\hline $\begin{array}{l}\text { ID. } \\
\text { VE }\end{array}$ & $\begin{array}{l}\text { Conec } \\
\text { ta... }\end{array}$ & $\begin{array}{l}\text { Sup. } \\
\text { total } \\
\left(\mathrm{m}^{2}\right)\end{array}$ & Pend Azimut & $\begin{array}{l}\mathrm{N}^{\circ} \text { vi } \\
\text { drios }\end{array}$ & $\begin{array}{l}\text { Coef. pérd. } \quad U+ \\
\text { U del vidr. } \\
\left(W / m^{2} K\right)\left(W / m^{2} K\right)\end{array}$ & $\begin{array}{c}\text { Sup. } \\
\text { mayor } \\
\text { exp. } \\
\text { solar } \\
\left(\mathrm{m}^{2}\right)\end{array}$ \\
\hline 1 & $\begin{array}{l}\mathrm{LU}_{3-} \\
\text { Ext. }\end{array}$ & 2,4 & $90^{\circ}$ & 1 & 5,82 & 0 \\
\hline
\end{tabular}

Nota: Datos técnicos sobre ventanas: ambientes que conecta, superficie total, pendiente, azimut, número de vidrios, coeficiente de pérdida $U$ del vidriado $(U)$, coeficiente de $U$ + postigos, superficie con mayor exposición solar.

\section{ANÁLISIS DE RESULTADOS}

\subsection{ESCENARIO BASE 1 (E1)}

Corresponde a la simulación de la Sala sin clases, para determinar el comportamiento en relación a la temperatura externa (Fig. 4). Esta situación representa la configuración térmica interna de los días sin actividad académica (incluyendo el receso de clases).

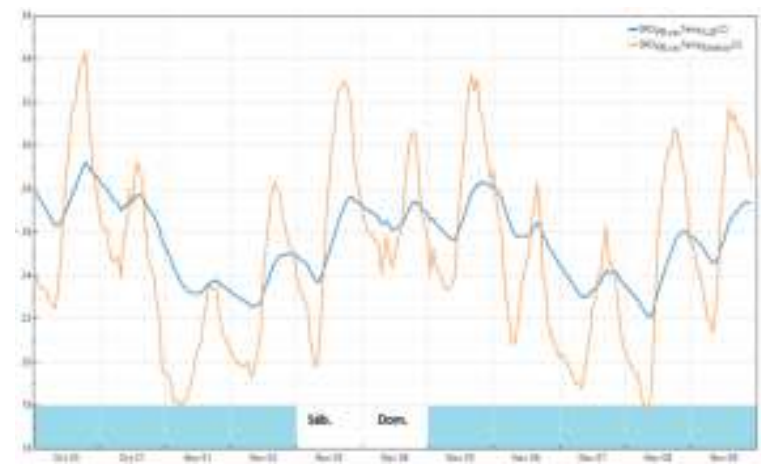

Fig. 4. Simulación del Escenario Base 1: temperatura interna de la Sala (color azul) y temperatura exterior (color naranja) correspondiente al periodo simulado 
De la simulación del escenario E1 se observa que:

- $\quad$ En el interior de la Sala, la temperatura media es $25,6^{\circ} \mathrm{C}$, la máxima de $29,2^{\circ} \mathrm{C}$ y la mínima de $22,1^{\circ} \mathrm{C}$; la amplitud térmica es $6,5^{\circ} \mathrm{C}$

- En el exterior, la temperatura media es $25,5^{\circ} \mathrm{C}$, la máxima es $34,3^{\circ} \mathrm{C}$ y la mínima 17,8 ; la amplitud térmica es $16,5^{\circ} \mathrm{C}$.

Conclusión: debido a su ubicación, el sombreado de árboles vecinos y edificaciones continuas al Norte y Este y Oeste y el tipo de techo, evitan las ganancias térmicas por exposición directa al sol.

\subsection{ESCENARIO BASE 2 (E2)}

Corresponde a la simulación de la Sala con actividad académica, iluminación completa, usando solamente los recursos didácticos básicos como proyector y la computadora del docente, con la asistencia de 22 alumnos, es decir en su capacidad máxima. Este escenario representa las situaciones de uso de la Sala cuando no existen aulas disponibles más que ésta, y no se requieren de equipos.

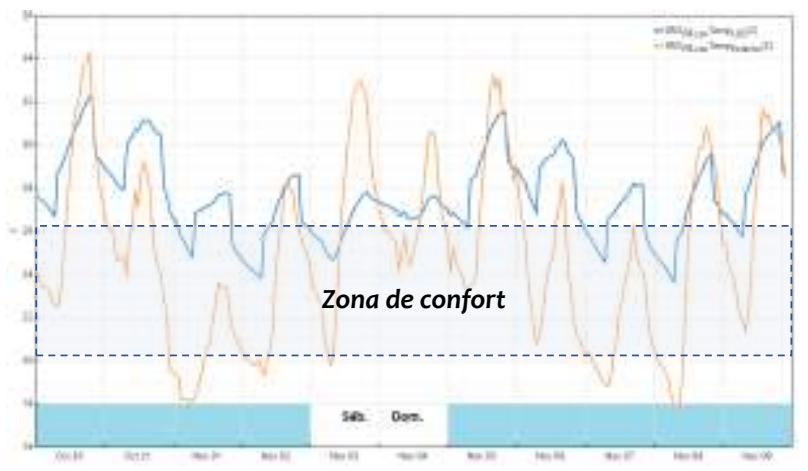

Fig. 5. Simulación del Escenario Base 2: gráfica generada con SIMEDIF con la temperatura interna de la Sala (color azul) y temperatura exterior (color naranja) correspondiente al periodo simulado

Del análisis de la simulación para el escenario E2 (Fig. 5), considerando solamente los días de clases (lunes a viernes) y los horarios de clases de 8 a 13 y de 15 a 21 hs, se observa que:

- $\quad$ en el interior de la sala, la temperatura media es $28,8^{\circ} \mathrm{C}$, la máxima de $32,3^{\circ} \mathrm{C}$ y la mínima $25,3^{\circ} \mathrm{C}$; la amplitud térmica es $7^{\circ} \mathrm{C}$

- el $55 \%$ de los días con clases, la temperatura interior supera a la exterior durante todo el día

- en los días en que la máxima exterior $\left(34,3^{\circ} \mathrm{C}\right)$ supera a la máxima interior, la diferencia oscila entre $1^{\circ} \mathrm{C}$ y $2^{\circ} \mathrm{C}$ (días: 30 de octubre, 5,8 y 9 de noviembre).

El análisis del confort del escenario E2 mediante el PMV muestra que $67 \%$ del tiempo el ambiente está ligeramente cálido, mientras que un 31\% está caluroso (Fig. 6).

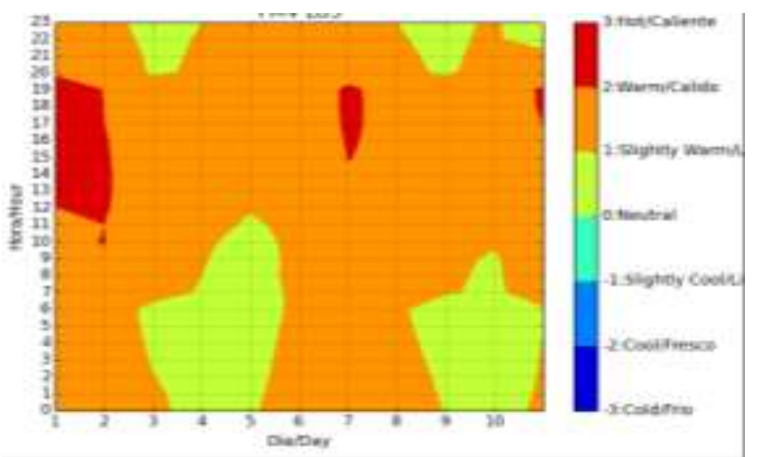

Fig. 6. PMV del Escenario Base 2, generado por SIMEDIF

Conclusión: durante los horarios de clases, aún sin el uso de computadoras, el ambiente presenta disconfort térmico (ligeramente caluroso a cálido).

\subsection{ESCENARIO BASE 3 (E3)}

Corresponde a la simulación de la Sala con actividad académica, iluminación completa, usando los recursos didácticos básicos como proyector y la computadora del docente, con el uso del 50\% de las computadoras y la asistencia de 50\% del alumnado (Fig. 7). Este escenario representa las clases de los cursos avanzados, en donde la cantidad de alumnos es reducida.

En la Fig. 7 se observan los resultados de la simulación del escenario E3:

- considerando solamente los días de clases y los horarios de clases establecidos, la temperatura media en el interior de la Sala es $29,2^{\circ} \mathrm{C}$, esto representa $0,4^{\circ} \mathrm{C}$ más que la media interior del escenario E2.

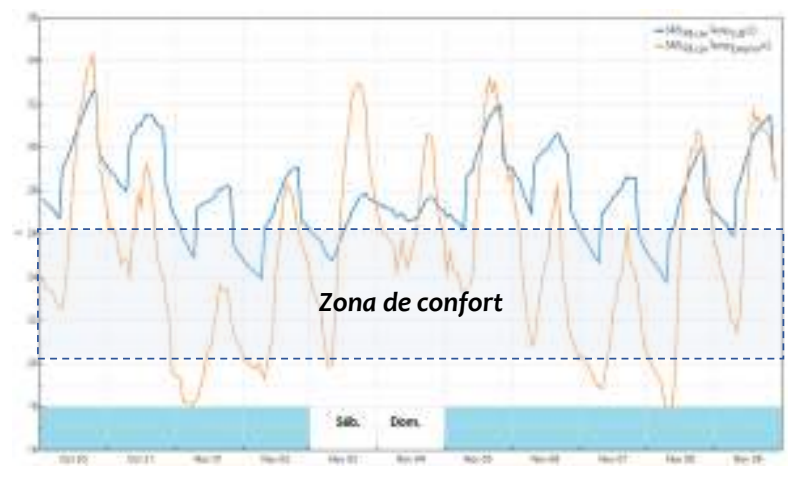

Fig. 7. Simulación del Escenario Base 3: gráfica generada con SIMEDIF con la temperatura interna de la Sala (color azul) y temperatura exterior (color naranja) correspondiente al periodo simulado

El análisis del confort del escenario E3 mediante el PMV muestra que el $64 \%$ del tiempo el ambiente está ligeramente cálido y el $34 \%$ está caluroso. 


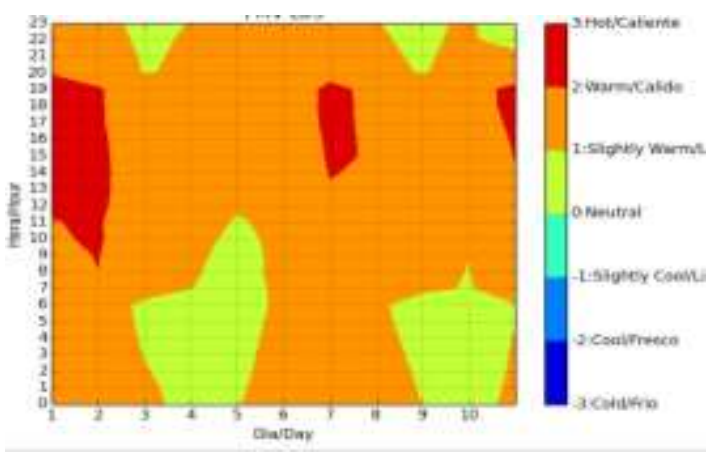

Fig. 8. PMV del Escenario Base 3, generado por SIMEDIF

Conclusión: El aumento de temperatura por actividad académica con el uso del 50\% de computadoras y 50\% del alumnado no genera grandes cambios térmicos en el ambiente de la sala comparado con el escenario E2. El ambiente E3 presenta disconfort térmico importante durante las horas de uso.

\subsection{ESCENARIO BASE 4 (E4)}

Corresponde a la simulación de la Sala con actividad académica, iluminación completa, usando los recursos didácticos básicos como proyector y la computadora del docente, con el uso del $100 \%$ de las computadoras y la asistencia del 100\% de alumnos. Este escenario representa las clases masivas de primer año.

Los resultados para el periodo simulado se observan en la Fig. 9:

- $\quad$ en el interior de la Sala, la temperatura media es $32,5^{\circ} \mathrm{C}$, la máxima de $35,9^{\circ} \mathrm{C}$ y la mínima $27,1^{\circ} \mathrm{C}$; la amplitud térmica es $8,8^{\circ} \mathrm{C}$

- el $100 \%$ de los días con clases, la temperatura interior supera a la exterior durante todo el día: las máximas internas oscilan entre $32^{\circ} \mathrm{C}$ y $36^{\circ} \mathrm{C}$

- la media interna de $\mathrm{E} 4$ es mayor en $3,7^{\circ} \mathrm{C}$ que la media interna del escenario E2.

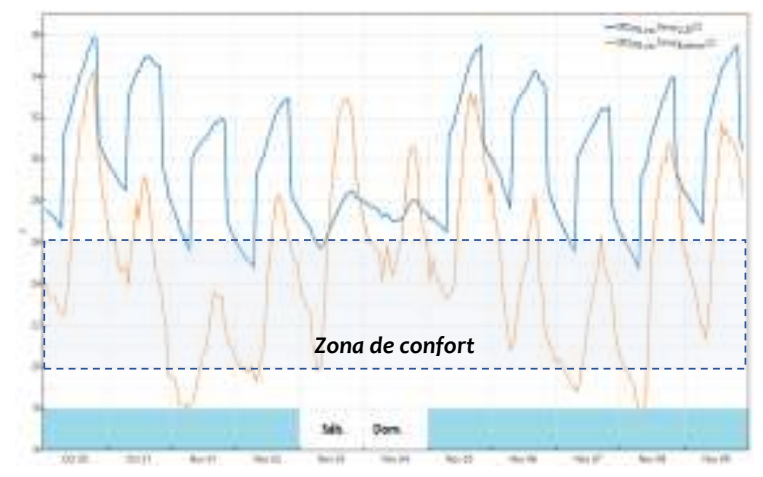

Fig. 9. Simulación del Escenario Base 4: gráfica generada con SIMEDIF con la temperatura interna de la Sala (color azul) y temperatura exterior (color naranja) correspondiente al periodo simulado
El análisis del confort del escenario E4 muestra que el $44 \%$ del tiempo el ambiente está ligeramente cálido, el $35 \%$ está caluroso y el $18 \%$ es caliente.

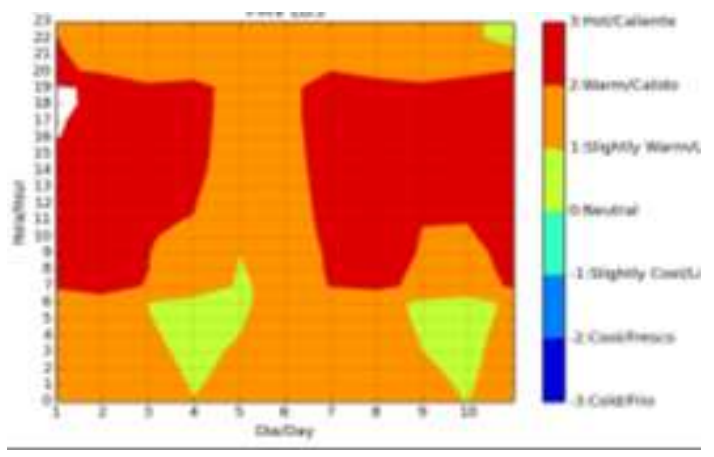

Fig. 10. PMV del Escenario Base 4, generado por SIMEDIF

Conclusión: las clases con el uso del 100\% de las computadoras y de alumnos se dan en un ambiente con condiciones de disconfort térmico muy alto (cálido a caliente). Para el escenario E4 resulta indispensable el uso de sistemas de refrigeración.

\subsection{ESCENARIO 5 (E5)}

Corresponde a la misma descripción del escenario E4 pero con el funcionamiento del equipo de aire acondicionado a $24^{\circ} \mathrm{C}$. Permite determinar la carga auxiliar necesaria para generar un ambiente confortable durante el dictado de clases.

En la Fig. 11 se grafica los resultados de la simulación y se observa que para lograr un ambiente confortable de 8 a 21 hs se requiere una carga diaria de refrigeración de aproximadamente -70kWh/día. El equipo de refrigeración debería poder extraer, como mínimo para este periodo $7 \mathrm{kWh}$.

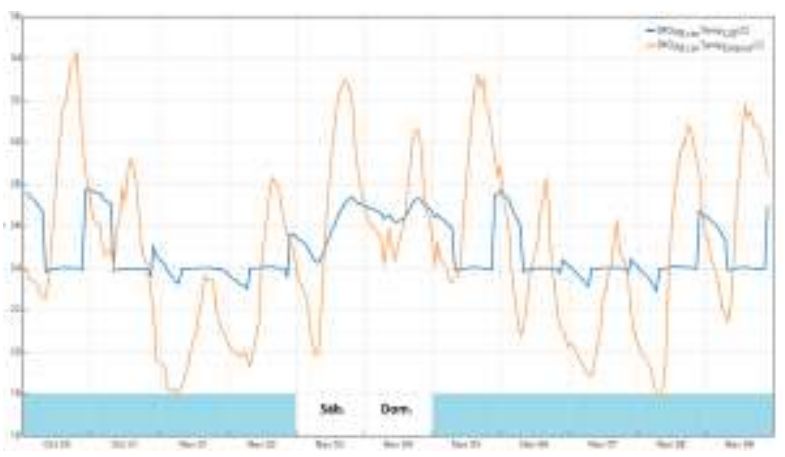

Fig. 11. Simulación del Escenario Base 5: gráfica generada con SIMEDIF con la temperatura interna de la Sala (color azul) y temperatura exterior (color naranja) correspondiente al periodo simulado

Conclusión: un equipo de aire acondicionado de 7 kWh cubriría los requerimientos de confort durante las horas de clases en periodos similares al estudiado. Para el dimensionado definitivo del equipo, deberían considerarse los días extremos en los que la 
temperatura exterior alcanza valores máximos absolutos.

\subsection{ESCENARIO X (EX)}

Se trata de un escenario-marco en el que se puede representar cualquiera de los escenarios bases (E1, E2, E3, E4), pero con temperaturas externas iguales o superiores a $40^{\circ} \mathrm{C}$ considerando solamente el rango horario de clases ( 8 a 13 y 15 a 21 hs).

El caso a aplicar corresponde a dos días consecutivos con altas temperaturas: 27 y 28 de octubre de 2019 , con temperaturas máximas de $43,8^{\circ} \mathrm{C}$ y $44,5^{\circ} \mathrm{C}$ respectivamente [8], ambas registradas a horas 17 . Suponiendo que en ambos días se desarrollan actividades académicas normales de 8 a 13 y de 15 a 21, se configuran los siguientes escenarios:

- E1X: simulación del E1 con temperaturas extremas

- E2X: simulación del E2 con temperaturas extremas

- E3X: simulación del E3 con temperaturas extremas

- E4X: simulación del E4 con temperaturas extremas.

TABLA V

Resultados de la Simulación en Condiciones Extremas Altas

\begin{tabular}{cccccccccccc}
\hline \multicolumn{4}{c}{ Temp.Amb } & E1X & \multicolumn{2}{c}{ E2X } & E3X & \multicolumn{2}{c}{ E4X } \\
Fec & $\begin{array}{c}\text { Med. Máx. } \\
\left({ }^{\circ} \mathrm{C}\right)\end{array}$ & $\begin{array}{c}\left.{ }^{\circ} \mathrm{C}\right) \\
\text { Int. }\end{array}$ & $\begin{array}{c}\text { Int. } \\
\left({ }^{\circ} \mathrm{C}\right)\end{array}$ & $\begin{array}{c}\text { Int. } \\
\left({ }^{\circ} \mathrm{C}\right)\end{array}$ & $\begin{array}{c}\text { Int. } \\
\left({ }^{\circ} \mathrm{C}\right)\end{array}$ & $\begin{array}{c}\text { Int. } \\
\left({ }^{\circ} \mathrm{C}\right)\end{array}$ & $\begin{array}{c}\text { Int. } \\
\left({ }^{\circ} \mathrm{C}\right)\end{array}$ & $\begin{array}{c}\text { Int. } \\
\left({ }^{\circ} \mathrm{C}\right)\end{array}$ & $\begin{array}{c}\text { Int. } \\
\left({ }^{\circ} \mathrm{C}\right)\end{array}$ & $\begin{array}{c}\text { Int. } \\
\left({ }^{\circ} \mathrm{C}\right)\end{array}$ \\
\hline $27 / 10$ & 36,6 & 43,5 & 33,0 & 34,7 & 35,1 & 37,2 & 35,7 & 37,9 & 38,2 & 40,8 \\
\hline $28 / 10$ & 37,5 & 44,5 & 33,4 & 35,2 & 35,9 & 37,9 & 36,6 & 38,7 & 39,4 & 41,8
\end{tabular}

Nota: temperaturas medias y máximas del ambiente externo y de interior de la Sala.

Los resultados de la simulación de los 4 escenarios extremos se registran en la TABLA V, indicando para cada caso las temperaturas internas media y máxima, todas superiores a los $33^{\circ} \mathrm{C}$.

Si se termostatiza el escenario $\mathrm{E} 4 \mathrm{X}$ para lograr un ambiente confortable de $24^{\circ} \mathrm{C}$ en el interior de la Sala durante el horario de clases, se requiere una carga diaria de refrigeración de -120kWh/día.

Algunas conclusiones importantes:

- las simulaciones en días con temperaturas extremas para los 4 escenarios muestran ambientes severamente desconfortables en la Sala de informática

- es indispensable un sistema de refrigeración que extraiga por lo menos $9 \mathrm{kWh}$ de energía para que las clases se den en un ambiente de confort higrotérmico
- la Sala dispone de un equipo que efectivamente puede responder a estas situaciones extremas.

\section{CONCLUSIONES}

- De acuerdo a la zonificación bioclimática de la Argentina (Norma IRAM 11603), Orán se encuentra en la zona IIb (clima cálido-húmedo). Presenta épocas muy calurosas, con temperaturas extremas que superan frecuentemente los $40^{\circ} \mathrm{C}$.

- En los últimos años, las temperaturas mayores a $30^{\circ} \mathrm{C}$ se han venido manifestando a partir del mes de setiembre, con valores extremos durante los meses de octubre y noviembre, afectando el ambiente en que se desarrollan las actividades durante el segundo cuatrimestre de clases en el ámbito universitario.

- En épocas estivales calurosas, las jornadas de clases en la Sede (y de cualquier institución) se tornan muy desconfortables sin la ayuda de ningún sistema mecánico de refrigeración. El uso de ventiladores ya no es suficiente, la solución práctica y rápida para el logro del confort térmico viene de la mano de los equipos de aire acondicionado. Cada vez se buscan que sean de mayor capacidad. Por consiguiente, la demanda energética aumenta notablemente y genera desabastecimiento año tras año.

La situación descripta en la Sala puede ser representativa de múltiples entornos de trabajo, en donde se usan computadoras, interactúan muchas personas y recurren a los equipos de aire acondicionado para el logro del confort térmico.

De la simulación se obtuvo:

- Una aproximación de cómo es el comportamiento térmico de este ambiente en diferentes escenarios, considerando las condiciones climáticas locales (las altas temperaturas y humedades).

- Los ambientes de disconfort y las condiciones en que los docentes y alumnos trabajan sin el uso de equipos de refrigeración.

- Se reconoce que los equipos de aire acondicionado son elementos necesarios para esta zona, pero si se combinara con el uso de estrategias bioclimáticas y de refrescamiento pasivo, se reduciría el tiempo de uso, y por consiguiente su costo. Estas acciones contribuirían al medio ambiente, porque se disminuiría las emisiones de gases de efecto invernadero generado por el sector energético. 


\section{AGRADECIMIENTOS}

Se agradece profundamente a la Dra. Silvana Flores Larsen, docente la Universidad Nacional de Salta, por su acompañamiento y asesoramiento en el desarrollo de este trabajo.

\section{REFERENCIAS}

[1] M. Beccali, V. Strazzeri, M.L. Germanà, V. Melluso and A. Galioto, "Vernacular and bioclimatic architecture and indoor thermal comfort implications in hot-humid climates: An overview", Renewable and Sustainable Energy Reviews, vol. 82, pp. 1726-1736, 2018.

[2] ONU (2019, Aug 14). United Nations, Department of Economic and Social Affairs, Population Division. World Urbanization Prospects 2018. Available: https://population.un.org/wup/Download/

[3] I. Ballarini and V. Corrado, "Application of energy rating methods to the existing building stock: analysis of some residential buildings in Turin", Energy and Buildings, vol. 41, no.11, 2009.

[4] I. Blasco Lucas, "Aportes de la arquitectura sustentable en el sector residencial sobre el balance energético-ambiental argentino", AVERMA, vol. 12, pp. 07.17-07.24, 2008. Available: http://www.cricyt.edu.ar/asades/modulos/averma/trabajos/2008/200 8-to07-a003.pdf

[5] WRI (2019, oct 20). WRI lidera la Iniciativa Edificios Cero Carbono para Todos lanzada en la Cumbre de Acción Climática de la ONU. Available: https://www.wri.org/news/2019/og/release-wri-leads-zerocarbon-buildings-all-initiative-launched-un-climate-action

[6] S. de la Rue Du Can and L. Price. "Tendencias sectoriales en el uso global de energía y las emisiones de gases de efecto invernadero", Política energética, vol. 36, no. 4, pp. 1386-1403,2008.

[7] C. Beck, J. Grieser, M. Kottek, F. Rubel and B. Rudolf. Characterizing Global Climate Change by means of Köppen Climate Classification, 2005.

[8] SMN (2019, oct 22). Servicio Meteorológico Nacional. Available: https://www.smn.gob.ar/

[9] CRC-SAS (2019, oct 30). Centro Regional del Clima del Sur de América del Sur. Available: http://www.crcsas.org/es/monitoreo_por_ciudades.php

[10] Weather Spark (2019, Aug 20). El clima promedio en San Ramón de la Nueva Orán. Available: https://es.weatherspark.com/y/28182/Clima-promedio-en-SanRam\%C3\%B3n-de-la-Nueva-Or\%C3\%A1n-Argentina-durante-todo-el$\mathrm{a} \% \mathrm{C}_{3} \% \mathrm{~B} 10$

[11] N. Sarmiento Barbieri, S. Belmonte, P. Dellicompagni, J. Franco y K. Escalante. Atlas de Radiación Solar de la Provincia de Salta. Salta, Argentina: Sistema de Información Geográfico Digital. Grupo Planificación Energética y Gestión Territorial. Instituto de Investigaciones en Energía No Convencional. CONICET- U.N.Sa., 2017.

[12] S. Flores Larsen (2019, Oct 30). SIMEDIF, Simulación térmica de $\begin{array}{lll}\text { edificios } & \mathrm{V} & \text { 2.0. Available: }\end{array}$ http://170.210.201.130/index.php/es/software/simedif-2-o-calculotermico-de-edificios/

[13] S. Flores Larsen y G. Lesino, "Modelo térmico del programa SIMEDIF de simulación de edificios", Energías Renovables y Medio Ambiente, vol. 9, pp. 15-24, 2001.

[14] A. Binda y G. Lesino, "Simulación computacional del comportamiento térmico de edificios para verano", en Actas de $12^{\circ}$ Reunión de ASADES, Buenos Aires, Argentina, 1987, vol. II, pp. 3.173.20 .

[15] A. Hernández y G. Lesino G., “Análisis de la performance térmica de un prototipo de vivienda liviana: monitoreo y simulación macrodinámica", Parte I, en Actas de la $16^{\circ}$ Reunión de ASADES, Tomo I, La Plata, Argentina, 1993, pp. 167-174.

[16] A. Esteves, J. Fernández, M. Basso, J. Mitchel y C. de Rosa, "Simulación térmica de edificios: aplicación de los modelos Quick y SIMEDIF", en Actas de la $17^{\circ}$ Reunión de ASADES, Rosario, Argentina, 1994, pp. 543-550.

[17] A. Beascochea y C. Filippín, "Un edificio solar pasivo para la Universidad Nacional de La Pampa. Dos años de resultados",
Avances en Energías Renovables y Medio Ambiente, vol.2, no. 1, pp. 5558, 1998.

[18] A. Hernández, S. Flores Larsen, N. Salvo y G. Lesino, "Simulación no estacionaria mediante SIMEDIF del ala oeste del edificio de Agronomía de la Universidad Nacional de La Pampa", Avances en Energías Renovables y Medio Ambiente, vol. 3, no. 2, pp. 8.113-8.116, 1999.

[19] A. Hernández y G. Lesino, "Simulación mediante SIMEDIF del comportamiento térmico de un prototipo de vivienda liviana construido en la Universidad Nacional de Salta", Avances en Energías Renovables y Medio Ambiente, vol. 4, no. 2, pp.8.29-8.34, 2000.

[20] C. Filippín y L. Marek, "Monitoreo higrotérmico, energético y socio ambiental de una Escuela Solar en la Provincia de La Pampa", AVERMA, vol. 8, no. 1, 2004.

[21] V. García, A. Iriarte, G. Lesino, S. Flores Larsen y C. Matías "Estrategias bioclimáticas para la producción de plantines de Nogal microinjertados", Energías Renovables y Medio Ambiente, vol. 18, pp. 37-44, 2006.

[22] A. Hernández, "GEOSOL: Una herramienta computacional para el cálculo de coordenadas solares y la estimación de irradiación solar horaria", Avances en Energías Renovables y Medio Ambiente, vol. 7, no. 2, 2003.

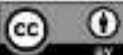

Los artículos publicados por TECNIA pueden ser compartidos a través de la licencia Creative Commons: CC BY 4.0. Permisos lejos de este alcance pueden ser consultados a través del correo revistas@uni.edu.pe 